\title{
Smartphone y redes sociales: una aproximación a los usos, vulnerabilidades y riesgos durante la adolescencia en España y Colombia
}

\section{Smartphone and social networks: an approach to the uses, vulnerabilities and risks during adolescence in Spain and Colombia}

\author{
MONTES-VOZMEDIANO, Manuel ${ }^{1}$ \\ PASTOR RUIZ, Yolanda ${ }^{2}$ \\ MARTÍN-NIETO, Rebeca ${ }^{3}$ \\ ATUESTA REYES, Juan D. ${ }^{4}$
}

\begin{abstract}
Resumen
Este artículo estudia la percepción que poseen los adolescentes sobre los usos, las vulnerabilidades y los riesgos de las redes sociales y el smartphone en España y Colombia. Para ello se han realizado diferentes grupos de discusión en ambos países. Se empleó el análisis de contenido clásico para estudiar el discurso. Entre los riesgos percibidos, destacaron el manejo de la imagen personal online y con menor importancia el contacto con desconocidos y personas indeseables.

Palabras clave: redes sociales; adolescentes; usos; riesgos

Abstract

This article studies the perception that adolescents have about the uses, vulnerabilities and risks of social networks and the smartphone in Spain and Colombia. To this end, different discussion groups have been held in both countries. Classical content analysis was used to study the discourse. Among the perceived risks, they highlighted the handling of personal image online and with less importance the contact with strangers and undesirable people.

key words: social media; adolescents; uses; risks
\end{abstract}

\section{Introducción}

Con la expansión del uso del smartphone en la última década, los medios sociales se han convertido en la herramienta básica de comunicación con los iguales y la predominante para acceder al consumo de contenidos audiovisuales entre los adolescentes.

El teléfono móvil constituye la principal pantalla a la que se exponen hoy en día (Del Barrio, 2014: 564). Su versatilidad, fácil uso y portabilidad hacen de este dispositivo el favorito para comunicarse y acceder a las diferentes redes sociales y a la mensajería instantánea.

\footnotetext{
${ }^{1}$ Profesor del Departamento de Ciencias de la Comunicación y Sociología de la Universidad Rey Juan Carlos. España. manueljavier.montes@urjc.es

2 Profesora del Departamento de Psicología de la Universidad Rey Juan Carlos. España. yolanda.pastor@urjc.es

${ }^{3}$ Profesora del Departamento de Ciencias de la Comunicación y Sociología de la Universidad Rey Juan Carlos. España. rebeca.martin@urjc.es

${ }^{4}$ Profesor asistente y Director del Programa de Diseño Industrial de la Universidad Católica de Pereira. Colombia. juan.atuesta@ucp.edu.co
} 
El presente artículo revisa el estado de la cuestión sobre el uso de las redes sociales y el teléfono móvil en la etapa adolescente y analiza las percepciones que poseen los adolescentes (Alonso-Ruido et al., 2015: 55) españoles y colombianos sobre los usos de los medios sociales y las vulnerabilidades a las que se exponen, así como el papel de la familia en su acceso a estas tecnologías, a partir de un estudio cualitativo llevado a cabo con grupos de discusión en ambos países.

Según los datos de los informes Net Children Go Mobile (Garmendia et al., 2016: 19) y TigoUne (2018: 12), más de tres cuartas partes de los adolescentes españoles y colombianos posee un smartphone y, entre los 16 y los 17 años, casi todos disponen de un dispositivo móvil de uso personal. La edad de acceso al primer teléfono inteligente de uso personal se va adelantando cada año (Garmendia et al., 2016: 21). Esto ha producido un cambio en el patrón de consumo de estos medios por parte de los adolescentes, caracterizado por un uso cada vez más intensivo en cualquier momento y lugar (Garmendia et al., 2016, 95; Sendín et al., 2014: 997), de modo que les resulta más difícil delimitar el tiempo diario que dedican a los medios sociales, afirmando estar continuamente conectados (Del Barrio, 2014: 564; Álvarez et al., 2019: 18).

Los adolescentes acceden a todo tipo de redes sociales y las combinan con el uso de aplicaciones de mensajería instantánea (Garmendia et al., 2016: 27; TigoUne, 2018: 15). A través de estos medios les llegan contenidos audiovisuales sin tan siquiera buscarlos. No obstante, el uso diferencial de estos medios ha sido poco estudiado. A pesar de que es frecuente que los adolescentes tengan perfiles abiertos en varias redes a la par, lo cierto es que suelen utilizar preferentemente una (Marcelino Mercedes, 2015: 56).

Además, la preferencia por una u otra red social parece estar cultural y/o contextualmente determinada. Mientras que en el 2009 los adolescentes españoles utilizaban Tuenti mayoritariamente, poco a poco fueron abandonando esta red, trasladándose al uso de Facebook. Este fenómeno ha recibido el nombre de migración en las redes sociales (Marcelino Mercedes, 2015: 50). Según diversos estudios (Almansa et al.,2013; Alonso-Ruido et al., 2015: 54; Marcelino Mercedes, 2015: 55), Facebook era la red social más utilizada por los adolescentes españoles entre 2009 y 2014. No obstante, parece ser que en los últimos años estamos asistiendo a una segunda migración de la población adolescente española hacia Instagram, así lo muestran los datos de audiencias, apertura de cuentas e incluso la presencia de publicidad y celebridades juveniles (Marcelino Mercedes, 2015: 68). Prades y Carbonell (2016: 32) estudiaron las motivaciones para el uso de Instagram con una muestra de 202 jóvenes entre 16 y 24 años. Encontraron que la principal motivación era la de observar y obtener información sobre lo que hacen sus contactos, seguido de la interacción social y la de almacenamiento. Además, interactuar lejos de la mirada de los adultos parece ser otro de los motivos que favorece el uso de Instagram frente a otras redes sociales (Madden et al., 2013). Cabe señalar que alrededor del 95\% de los adolescentes y jóvenes entraban en Instagram a través de su teléfono móvil, siendo las principales actividades que realizaban: mirar lo que hacen sus contactos (43\%), explorar la página de inicio (38\%), mirar el perfil de un personaje (33\%) y colgar fotografía y vídeos (35\%) (Prades \& Carbonell, 2016: 35).

Mientras en Colombia el informe TigoUne (2018: 15) pone de manifiesto que Facebook sigue siendo en la actualidad la red social más utilizada durante la adolescencia. Un estudio cualitativo que compara el uso de Facebook entre los adolescentes españoles y colombianos muestra que en ambos países necesitan formar parte de la red y presentarse de la forma más original posible, ambos utilizan un lenguaje propio con muchas incorrecciones gramaticales y suben fotos Ilamativas (Almansa et al., 2013: 133-134). Corredor et al., (2011: 4548) confirman que las redes sociales en Colombia se perciben por los adolescentes como una herramienta de interacción social informal para interactuar con otros en un espacio de "baja presión y bajas pretensiones", como una forma de crear un sentido de comunidad y "mantener relaciones constantes en un contexto de alto cambio y movilidad".

Otra de las plataformas de frecuente uso para los adolescentes es YouTube. En un trabajo de revisión sobre el 
uso de esta plataforma en la adolescencia (García et al., 2016: 65-66) se encuentra que la mayoría la utiliza para acceder a contenidos audiovisuales de todo tipo, compartirlos y utilizar la herramienta "me gusta", siendo muy pocos los jóvenes que publican sus propios vídeos. No todos ellos suelen tener un perfil en YouTube, dado que no es imprescindible para acceder a los materiales. Los contenidos que habitualmente consumen en YouTube son vídeos divertidos, sobre hobbies o sobre ámbitos que les interesan, sobre cómo hacer algo o aprender una nueva habilidad, información sobre películas o programas de TV, sobre personajes famosos y youtubers e incluso sobre aspectos educativos (García et al., 2016: 69). Las publicaciones realizadas por los youtubers obtienen un amplio apoyo en las redes sociales, comparadas con las de cualquier otro agente emisor (Montes-Vozmediano et al., 2018: 67). A través de YouTube los adolescentes contactan con otras personas con sus mismos gustos e intereses, expresan sus preferencias y encuentran amigos online (Lenhart et al., 2015). YouTube suele ser frecuentado por adolescentes de todas las edades, pero cabe señalar que los más pequeños o incluso en la infancia acceden a sus contenidos. Algunos estudios empiezan a aventurar que podrían contribuir a la formación de la identidad en este periodo (Pérez et al., 2018: 67). En un estudio con preadolescentes sobre la influencia social de los youtubers concluyeron que estas celebridades constituyen referentes para los más pequeños que les otorgaban un valor como figura de entretenimiento. Los preadolescentes desean formar parte de la cultura digital juvenil que representa a los youtubers; no obstante, se muestran críticos con las opiniones poco respetuosas de estos personajes públicos y perciben su interés comercial centrado en ganar audiencia (AranRamsport et al., - 2018: 78). Esta influencia de los youtubers no parece repercutir en su formación ya que según un estudio son la tipología de autores que en menor medida aporta contenidos de prevención o formación (García \& Montes 2020: 13). A los adolescentes les gusta realizar vídeos y que estos sean vistos y valorados, si bien reconocen sus limitaciones como creadores de este tipo de material (De la Fuente Prieto et al.,-2019: 183184).

Según estudios previos, WhatsApp es empleado por el $88 \%$ de los adolescentes, para quedar con amigos o simplemente para contactar con ellos, compartir y hacer fotos y vídeos, acceder a enlaces web que reciben, bajar juegos e imágenes y también para contactar con la familia (Del Barrio, 2014: 569). El 84\% de los adolescentes entre 12 y 17 años consideran que esta aplicación es imprescindible, interesante y divertida. Muchos de ellos además $(56,7 \%)$ se consideran bastante o muy enganchados a esta aplicación, siendo las chicas las que realizan en mayor medida ese tipo de valoraciones (Del Barrio y Ruiz, 2017: 27-28). El uso de WhatsApp se ha generalizado en este colectivo y algunos autores remarcan que su uso es incluso más intensivo y generalizado que las redes sociales (Del Barrio y Ruiz, 2017: 29).

Los estudios actuales inciden en que no hay diferencias entre chicos y chicas en la frecuencia, tiempo dedicado y horario en su conexión a internet (Golpe et al., 2017: 139; Rial et al., 2014: 645). En ambos el uso de internet, las redes sociales o la mensajería instantánea cumple básicamente una función social. Las diferencias de género aparecen en cómo usan estos medios. Mientras los chicos suelen dedicar más tiempo a jugar online, las chicas comparten mayor información con sus contactos (Golpe et al., 2017: 139). También se diferencian en el contenido que consultan a través de las redes sociales, mientras los varones muestran más interés en la información deportiva, las chicas se decantan por la música o por seguir a personas famosas en redes sociales (Pastor et al., 2019: 1005). Varios son los autores que inciden además en que las chicas expresan sus emociones, sentimientos y comparten contenidos íntimos en mayor medida (Alonso-Ruido et al., 2015, 55; Chóliz et al., - 2009: 87-88; Sabater \& Fernández 2015: 240-246). Por ese motivo se considera que están más expuestas (Alonso-Ruido et al., 2015: 56). Los estudios también informan de un mayor uso de las chicas del smartphone y el WhatsApp (Golpe et al., 2017: 139).

Con respecto a la seguridad y privacidad en las redes sociales, los estudios ponen de manifiesto cierto desconocimiento por parte de los adolescentes sobre los mecanismos que éstas ofrecen para protegerse y/o gestionar la privacidad, así como una falta de conciencia sobre los problemas que pueden derivarse de un mal uso 
de estos mecanismos (Rodríguez \& Magdalena 2016: 25). Mientras que algunos trabajos inciden en que en los primeros años de la adolescencia es cuando se asume más riesgos y que cuando se empieza a tener experiencias negativas en la adolescencia media aprenden a gestionar mejor su privacidad en las redes (Merritt 2008: 8-10); otros afirman que se produce una disociación entre las experiencias negativas y la percepción de riesgo en la adolescencia; y que por el contrario a pesar de las experiencias desagradables en general los adolescentes se sienten seguros y poseen una alta sensación de control en este medio (De-Frutos \& Marcos 2017 93). Lo cierto es que alrededor del $40 \%$ de los adolescentes se muestran preocupados por la seguridad y confidencialidad de las redes sociales, siendo mayor el porcentaje de chicas, en comparación con sus coetáneos, las que se manifiestan preocupadas (Rial et al., 2014: 650).

Con respecto a las medidas de seguridad que adoptan, el 66\% afirma tener su perfil en privado solo para sus amigos (De-Frutos \& Marcos 2017 91). Tan solo entre el 15 y el 25\% de los usuarios adolescentes de las redes sociales y mensajería instantánea se han leído las políticas de privacidad de las mismas. Muchos de ellos afirman que solo agregan a personas de su confianza y que realizan cambios en las opciones de privacidad de las redes. No obstante, son pocos los que cuidan el cambio de contraseñas con cierta regularidad y por el contrario utilizan la misma contraseña en distintas redes (Rodríguez \& Magdalena, 2016: 34-36). Otros datos proporcionados sobre los adolescentes colombianos muestran que el $79 \%$ de ellos afirmaban saber bloquear a alguien en una red social, el 51\% sabían cambiar los ajustes de privacidad de una red social pero sólo el 39\% sabría gestionar los ajustes de privacidad de su navegador (TigoUne, 2018: 16). Cabe resaltar, no obstante, que estudios observacionales sobre el uso de cuentas y perfiles de adolescentes españoles y colombianos ponen de manifiesto que el empleo de medidas protectoras no es tan generalizado como afirman, suelen agregar contactos de personas que no conocen personalmente en base a la apariencia en una fotografía o porque tienen amigos en común (Almansa et al., 2013: 131). En general ambos colectivos se sobreexponen en las redes. Cabe señalar que el nivel de sobreexposición era mayor en el caso de los jóvenes españoles, éstos utilizaban en mayor medida su auténtico nombre en los perfiles casi de forma mayoritaria y revelaban más datos personales que sus coetáneos colombianos (Almansa et al., 2013: 134).

A pesar de la ingente cantidad de estudios que se han realizado sobre los riesgos del uso de internet, redes sociales y smartphone en la adolescencia (para una revisión véase Martínez et al., (2019): 257-268), pocos trabajos informan de la percepción de los riesgos por este colectivo. Uno de los primeros trabajos realizados con jóvenes españoles ponía de manifiesto que éstos no percibían graves riesgos del uso de internet, se sentían identificados como grupo con la tecnología y seguros a la hora de manejar situaciones con desconocidos. En contraposición percibían riesgos importantes para adolescentes o niños menores que ellos (Espinar \& López, 2009: 17). En un estudio realizado con adolescentes gallegos, se ha encontrado que los adolescentes tienden a percibir que otras personas de su edad pueden hacer un uso poco responsable y arriesgado de internet, mientras ellos mismos se excluyen de esos riesgos, se perciben con apego al uso de los medios y la tecnología, pero con baja probabilidad de dependencia. A mayor edad muestran una mayor conciencia de estos riesgos (Rial et al., 2014: 652), aunque el estudio realizado por Tejada Garitan et al., 2019: 128-129) informa que la percepción del riesgo es muy baja ya a los 11 años. Otro trabajo con adolescentes españoles también informaba de que se manifestaban conocedores de los riesgos del uso de internet, aunque no se percibían a sí mismos con riesgo de dependencia y no veían como amenazantes las descargas ilegales o los contenidos inapropiados que pueden encontrar en la red. Por el contrario, percibían como preocupante cualquier acción que pudiera dañar su imagen pública o los contactos con desconocidos, aunque se sentían con capacidad para saber responder a esta última situación. Muchos manifestaron haber recibido amenazas, chantajes o mensajes insultantes, situaciones que calificaron como desagradables, pero no las percibían como una gran amenaza o riesgo (Martínez et al.,2013: 124-125). De hecho, parece que hay mayor consciencia del riesgo en las redes entre los adolescentes de 16-17 años por las experiencias negativas vividas que por un control parental riguroso (Pastor et al.,(2019: 1005). En 
un estudio con adolescentes madrileños encontraron que aquellos menores que presentaban mayor percepción de riesgo eran los que presentaban mayores habilidades para protegerse online, manifestaban mayor intervención educativa de sus progenitores y mostraban unas prácticas más saludables en general. En contraposición aquellos menores con baja percepción de riesgo online manifestaban actitudes negativas hacia el control de los padres y profesores, no los percibían como fuentes de apoyo o ayuda y tampoco los reconocían como figuras de autoridad (Ramos et al., 2018: 77-78).

Aunque los adolescentes no se perciben en general a sí mismos como dependientes del smartphone y las redes sociales, los estudios han puesto de manifiesto que en general calificaban de molesto o muy desagradable quedarse sin conexión a internet (Martínez et al., 2013: 117). Esta tendencia ha recibido el nombre en la actualidad de "miedo a perderse algo" o FOMO. Los estudios ponen de manifiesto que el FOMO es un correlato de malestar emocional y de un uso problemático tanto del móvil como de las redes sociales (Gil et al., 2015: 80; Santana-Vega et al., (2019: 42-43).

Acerca del papel de los progenitores, los estudios parecen coincidir en la escasa supervisión parental del uso y acceso de los adolescentes a las redes sociales (Alonso-Ruido et al., 2015; Giménez et al., 2017: 534; Rial et al., 2014: 651). Entre las estrategias más utilizadas para la supervisión se encuentran: la realización de preguntas directas, el límite temporal y la revisión del historial. Menos usadas fueron el acceso conjunto y registrar el ordenador (Giménez et al., 2017: 543).

El control parental es percibido por los adolescentes como "una forma de inmiscuirse en su vida social, en su identidad individual y de grupo, interviniendo en un aspecto íntimo de expresión y contacto -no físico- con su grupo de iguales" según un estudio comparativo entre adolescentes colombianos y españoles (Cantón Mayo et al., 2019: 195) por eso, cada vez es más común que, durante la adolescencia, empiecen a utilizar dispositivos con acceso a Internet fuera del control de los padres (García del Castillo et al., 2019: 176). Especialmente ocurre cuando el control parental se realiza "sin afecto, sin comunicación y sin tolerancia", lo que puede provocar indisciplina, evasión y adicciones (Malander, 2019: 38-39).

Un trabajo realizado con estudiantes de secundaria ofrece una mayor tasa de supervisión "familiar" (70\%) del uso de internet. En este caso se informa que la supervisión es llevada a cabo tanto por ambos progenitores como por los hermanos mayores. En este trabajo las chicas informaron recibir una mayor supervisión que los chicos. Asimismo, la supervisión disminuía a medida que aumentaba el curso académico (Giménez, Luengo \& Bartrina, 2017: 543).

Según informan los adolescentes en otro estudio con población gallega, más de la mitad de los padres y las madres no realizan ningún tipo de control de su uso, ni ponen límite alguno. Y el 77,9\% informan no haber tenido nunca o casi nunca una discusión con ellos sobre el uso de internet (Rial et al., 2014: 647). La falta de control se debe, en ocasiones, a la brecha digital que supone para algunos progenitores la ausencia de conocimientos y habilidades entorno a las tecnologías que les aleja, en ocasiones, de poder ejercer una autoridad comprometida y responsable respecto al uso que sus hijos hacen de ellas (López-Sánchez \& García del Castillo 2017: 117), si bien el concepto de tecnología no debe limitarse exclusivamente a la destreza con la que se manipula un artefacto sino a tener un pensamiento tecnológico (Atuesta Reyes, 2014: 73). En un estudio centrado en adolescentes colombianos y sus progenitores se observa además que esta brecha, afecta a las interacciones familiares provocando "distanciamiento y resistencia, abriendo paso a relaciones paterno filiales conflictivas" (Moreno López et al., 2017: 6-17). Este mismo estudio evidencia cómo los conocimientos de los adolescentes pueden aprovecharse para desarrollar planes de alfabetización digital donde faciliten la entrada a sus padres en el uso de las redes sociales. 


\subsection{Objetivos}

Los objetivos que han guiado esta investigación son los siguientes:

Averiguar qué redes sociales utilizan los adolescentes y con qué finalidad las emplean.

Conocer cómo perciben las diferentes redes sociales y la mensajería instantánea.

Entender cómo usan y perciben el smartphone.

Averiguar cuáles son los principales riesgos del uso de las redes sociales y la mensajería, desde su punto de vista.

- $\quad$ Saber qué estrategias utilizan para protegerse.

- $\quad$ Percepción de los jóvenes sobre el papel de la familia en el acceso a las redes sociales.

- Comparar a los adolescentes españoles y colombianos en estos aspectos.

\section{Metodología}

La metodología aplicada en esta investigación, que constituye la primera fase del proyecto enunciado al inicio del artículo, es de carácter cualitativo y se sustenta en la técnica del grupo de discusión. Partiendo de la investigación llevaba a cabo en España se considera la necesidad de conocer si los patrones de uso adolescente, su percepción del riesgo, sus estrategias de prevención y el papel familiar coinciden con los que tienen los adolescentes colombianos, iniciándose el acercamiento a esta realidad con la misma técnica.

La estrategia para la definición de los grupos de discusión inicial se configuró tras varias propuestas elaboradas por un equipo de investigadores (Martín Nieto et al., 2017: 421-423). En la presente investigación se han contemplado los criterios de representatividad del muestreo cualitativo que se consiguen "desde la comprensión de la naturaleza del objeto social, de las propiedades y características de las relaciones sociales estructurales relevantes para la investigación", tal y como manifiesta Mejía Navarrete (2000: 167-168). Las categorías o ejes a tener en cuenta para el diseño de la muestra estructural son los siguientes: la edad (13-17 años) y el sexo, el nivel educativo (Educación secundaria obligatoria ESO, Formación profesional FP y Bachillerato), el tipo de centro (público, privado y concertado), zona geográfica (norte, centro, sur) y nivel socioeconómico (alto, medio, bajo).

Las edades de los adolescentes que participaron en los grupos de discusión en España estaban comprendidas entre los 13 y 17 años. Se consideró pertinente evitar que coincidieran en un mismo grupo de discusión, menores con una diferencia que excediera los tres años ya que, seguramente, su percepción de las temáticas sería diferente y la opinión de los mayores podría coartar a los más jóvenes. Así pues, se planificaron dos grupos de discusión con menores de 13-15 años y otros dos con menores de 16-17 años.

Con objeto de captar la unidad discursiva y teniendo en cuenta todas estas categorías se diseñaron cuatro grupos de discusión con población española de la Comunidad de Madrid con los siguientes perfiles: 1) edad 13-15, zona norte, nivel socioeconómico alto, centro privado, ESO; 2) edad 13-15, zona sur, nivel socioeconómico mediobajo, centro público, ESO; 3) edad 15-17, zona centro, nivel socioeconómico medio, centro público, FP; y 4) edad 15-17, nivel socioeconómico medio, zona centro-sur, Bachiller. Cada uno de ellos formados por ocho menores y con paridad de género (cuatro chicos y cuatro chicas). En España, los centros colaboradores estaban ubicados en diferentes puntos de la Comunidad de Madrid y eran de diferente titularidad (pública, concertada y privada). En Colombia, se llevó a cabo un grupo de discusión procedente de un centro colaborador situado en la ciudad de Pereira, ubicada en el departamento de Risaralda. El grupo de discusión realizado en Colombia, con menores de 16-17 años, se ajusta al perfil de los jóvenes del cuarto grupo.

Los tópicos concretos introducidos por los investigadores en los grupos fueron los siguientes: 1) uso diferencial de las redes sociales y WhatsApp, 2) uso del smartphone, 3) percepción de los riesgos, 4) estrategias para protegerse y 5 ) el papel de la familia. 
Se llevó a cabo la trascripción literal del discurso de los adolescentes en cada uno de los grupos de discusión. Para el análisis de los textos producidos, se utilizó la técnica de análisis de contenido clásico. Al tratarse de un estudio con menores, cabe mencionar que se cumplieron escrupulosamente los protocolos de protección de datos e identidades, los compromisos relativos a las normas éticas de investigación así como los requerimientos legales imprescindibles para realizar un proyecto de investigación de esta índole. Se obtuvo el visto bueno del Comité de Ética de nuestra institución previamente a la puesta en marcha el estudio.

\section{Resultados}

A tenor del análisis de la información conseguida mediante los grupos de discusión se han definido los siguientes hallazgos que se muestran distribuidos por bloques.

\subsection{Redes sociales: usos y percepciones}

Entre los adolescentes, ya sean españoles o colombianos, el uso de Instagram se ha generalizado. Los grupos de discusión han puesto de manifiesto que la capacidad de esta red social de integrar las posibilidades que tenían otras redes (como las "historias", que eran uno de los rasgos definitorios de Snapchat, una red en desuso actualmente, mientras que están en auge otras como Tik Tok) la ha elevado a un lugar de preferencia entre el público joven. Los adolescentes manifiestan que, si bien en sus inicios Instagram parecía que servía solo para subir fotos, ahora les permite, además de publicar sus imágenes preferidas y compartirlas con los demás, estar al día de lo que hacen sus amigos y seguidores, enviar mensajes privados (textos, audios, etc.), socializar con los demás usuarios, publicar historias de lo que hacen, crear grupos específicos con algunos seguidores, en definitiva, aúna lo que más les gusta o valoran en una única red social. Entre los adolescentes colombianos que participaron en el grupo de discusión aparecían otras redes como alternativas o preferidas (como Facebook o Pinterest) pero todos tenían un perfil de Instagram, aunque no la considerasen su red predilecta.

Entre los principales motivos de uso de las redes sociales sitúan el entretenimiento como el primero de ellos, motivo por el cual no sitúan WhatsApp entre las redes sociales. No obstante, en su discurso el término "entretenimiento" no queda directamente asociado al de "diversión", sino que, más bien, lo que persiguen, y así lo matizan, es "evitar el aburrimiento".

Si quisiéramos segmentar los usos diferenciados de cada una de ellas, todavía es posible marcar ciertas diferencias. WhatsApp claramente queda asociada con la mensajería instantánea, se utiliza fundamentalmente para estar en contacto con las amistades, consultar los estados de la gente y la posibilidad de realizar llamadas o videollamadas (especialmente cuando están conectados a una red wifi y no tienen que consumir sus datos). Facebook era catalogada por los adolescentes españoles como una red para personas "mayores", que es utilizada por otras generaciones (padres, tíos, hermanos mayores, etc.) por lo que no la conocían muy bien pero sí tenían claro que no era para ellos e incluso alguno veía probable hacerse un perfil en el futuro, "cuando se haga mayor". En contrapartida, los adolescentes colombianos no compartían esa visión de Facebook. Muchos jóvenes colombianos manifestaron su predilección por esta red social pues les parecía que era la que "tenía más contenido", comentaron una variedad de temas o asuntos que podían seguir a través de esta red social y algún motivo también de entretenimiento para seguirla, como el uso de los memes.

Instagram es considerada, en líneas generales, como la red con un uso más versátil entre los adolescentes españoles y colombianos participantes en este estudio, pues colma la mayor parte de sus expectativas e incluso les permite estar informados. Algunos adolescentes españoles solo recurren a otras redes si no les queda más remedio (por ejemplo, a sus padres no les pueden llamar por Instagram si no tienen un perfil en dicha red).

Con respecto a los usos diferenciales de las redes una adolescente española lo describía así: "en Instagram pones tu felicidad, en Twitter tus penas y en WhatsApp lo cuentas". En este sentido, los adolescentes coinciden en 
señalar que en Instagram la gente suele ocultar sus verdaderos sentimientos y finge que lo está pasando bien y que todo es fantástico cuando la realidad no siempre lo es, "intentas aparentar que estás bien". Utilizan Twitter, a nivel de comunicación, además de para informarse, para expresar sus quejas, criticar a algún personaje público (compartiendo los hashtag) y para enviar mensajes a modo de indirecta a algún contacto para que se sientan aludidos, por ejemplo "que mal amigo es alguien que...". Finalmente, emplean WhatsApp de un modo más personal, para contactar con amigos y ver qué es lo que les pasa o cómo están en realidad.

Los adolescentes manifestaban seguir a personajes de referencia (los conocidos como influencers o youtubers) en sus redes sociales. Los motivos que exponían eran muy variados en este caso, a algunos los siguen para estar al tanto de los contenidos novedosos que promocionan o porque les entretiene, en otras ocasiones exponían que gracias a ellos se difunden con mayor facilidad ciertas denuncias de actividades ilícitas (por ejemplo, si una influencer denuncia que alguien sube vídeos de maltrato animal sus seguidores se hacen eco y consiguen que se retiren esos vídeos). Algunos exponían también que siguen a algún influencer reciente, sin un gran volumen de seguidores, porque es amigo o conocido suyo o de un tercero, con el fin de apoyarle y que sea más relevante.

La consulta de las redes sociales permite a los jóvenes estar al día de lo que hacen sus contactos, ya sean personas de su entorno más cercano o famosos, pero también conocer los hechos noticiosos (Vizcaíno et al., - 2017: 220), si bien para esto último no se ha podido definir una red social concreta ya que su consulta de la información de actualidad no parece seguir unos patrones homogéneos.

\subsection{Vulnerabilidades y percepciones de las redes sociales}

Uno de los riesgos más frecuentes en el uso de las redes sociales en la adolescencia es mantener contactos con desconocidos. Así pues, tal y como expresaban durante los grupos de discusión, es habitual que los adolescentes admitan entre sus contactos o seguidores los perfiles de personas que no conocen en la vida real. Esto es un hecho que perciben como normal. Una de las ventajas que observan en las redes sociales es que permiten "conocer gente nueva" y así aparecía en todos los grupos de discusión.

Con respecto al contacto con gente desconocida, incluso los adolescentes que aparentemente son más restrictivos en sus hábitos para admitir nuevos seguidores, manifiestan que admiten entre sus contactos a personas que conocen directamente, pero también a contactos de sus conocidos sin saber si ellos han empleado el mismo criterio. De este modo se crea una brecha en su seguridad que explica con claridad uno de los jóvenes: "si yo acepto a una persona que yo no conozco porque tenía ciertos amigos en común y yo ya la he aceptado porque tenía ciertos amigos en común, esa persona también puede haber aceptado a todas esas personas sin conocerlas".

En este sentido, sitúan a WhatsApp en una posición distinta a la de las otras redes sociales, la consideran mucho más segura que el resto de redes sociales, ya que la perciben como un entorno más privado que solo te pueden contactar si tienen tu número de teléfono móvil, mientras que en las otras redes pueden acceder a tu usuario $\sin$ ese requisito. Esto supone que los contactos del entorno cotidiano en el mundo real suelen estar replicados en WhatsApp (familiares, amigos o compañeros de clase) mientras que en las otras redes sociales tienen mayor presencia otros perfiles (amigos virtuales, influencers, conocidos o ligues, famosos a los que siguen y también desconocidos que engrosan su cuenta de seguidores).

No obstante, la principal preocupación durante la adolescencia en las redes sociales no son los desconocidos, sino el manejo de su imagen pública. Los adolescentes consideran que uno de los factores de riesgo inherentes a las redes sociales es el de someterse a un juicio público y la tensión que les genera si sus publicaciones son apoyadas o criticadas. Así lo expone un joven al afirmar que "es algo que siento que preocupa a los papás, pues tú estás metido dos, tres horas y ves muchos comentarios si subes una foto y escriben algo o no te dan un like al minuto y te sientes mal", concluye. 
En esta misma línea los adolescentes españoles comentaban que el modo en que podía afectar una crítica dependería de lo formada que se tuviera la personalidad y de quién era el agente de la crítica, si bien estaban de acuerdo en que esa crítica pública (por ejemplo, comentarios negativos sobre una foto que uno comparte) era algo que, con mayor o menor intensidad, les afecta a todos, incluso a los que dicen que eso no les importa. Entienden que cuando recibes comentarios negativos al final "te acabas rallando y de verdad piensas algo que no es, y a lo mejor acabas borrando la foto o dejando de subir cosas". Hacen hincapié, además, en que esos comentarios o críticas, al quedar escritos, trascienden en el tiempo y pueden cobrar protagonismo en otra ocasión.

Para los adolescentes colombianos la inseguridad en las redes sociales quedaba, en primer lugar, directamente ligada a las propias actitudes de los usuarios. El primer peligro se situaba en los contactos que uno permitía agregar y, después, en lo que cada cual publicaba y además criticaban la sobreexposición: "hay personas que comparten todo lo que hacen, absolutamente todo, y entonces uno tiene que ver hasta qué punto eso es bueno" afirma una joven, "si uno se va a postear todo lo que uno hace pues obviamente está corriendo peligro" se muestra contundente otra adolescente colombiana.

También son conscientes que otros usuarios pueden contactar con ellos, incluso sin tenerlos agregados, por ejemplo, les pueden enviar un mensaje. También han expuesto algunas pistas sobre cómo se puede detectar que ciertos perfiles son fraudulentos: "tú metes y como que ya sabes que es un perfil falso, la foto de un supermodelo, con diez amigos no más, y nada pues ya es como que es muy obvio que es falso". Cuando el intento de contacto por parte de desconocidos se vuelve desagradable (varias jóvenes manifiestan haber recibido fotos o vídeos con contenido sexual y entienden que es algo frecuente, "es algo que se ve mucho", dicen) su principal medida es el bloqueo del usuario. En la mayor parte de los casos este bloqueo suele ser suficiente para dejar de recibir mensajes de ese usuario.

Los adolescentes españoles tienen también en este aspecto una línea similar tanto de pensamiento como de actuación. Cuando les escribe o contacta con ellos alguien que desconocen y de un modo que no les agrada le bloquean y afirman que es una solución eficaz: "a mí sí que me ha hablado gente extraña, pero le bloqueo y ya está. Al final eso como que está normalizado", afirma un adolescente español. También han expresado haber ido un poco más lejos en alguna ocasión, por ejemplo, denunciando el perfil de algún usuario en redes sociales como Instagram.

Cabe reseñar que, en todos los grupos de discusión, se ha percibido que las chicas son las destinatarias más frecuentes de los intentos de desconocidos por entablar algún tipo de relación con ellas y se han mostrado más participativas en los grupos comentando circunstancias variadas por las que han tenido que tomar medidas con los perfiles de algún usuario.

Otro de los peligros que exponen los adolescentes colombianos es el referido a la manipulación de las imágenes o los vídeos que permiten las actuales tecnologías de modo que cualquier fotografía o audiovisual que uno comparta en sus redes sociales puede ser modificado con fines maliciosos. Consideran que estas manipulaciones de fotos o vídeos de una adolescente así como iniciar una conversación con un desconocido, son acciones que las comprometen y más peligrosas para la mayor parte de las menores de lo que ellas perciben: "muchas niñas caen en eso por un simple piropo, por decirlo así. Y por eso muchas veces las niñas salen violadas, secuestradas y también, en algún aspecto, lo utilizan indebidamente para pedirles plata, extorsionar a las personas", expone otra adolescente colombiana.

\subsection{Smartphone: usos y vulnerabilidades}

Es habitual emplear el smartphone de uno de los padres para que el menor tenga su primer acercamiento al entorno digital como usuario individual, para lo que existen diferentes fórmulas como puede ser la creación de 
un grupo de WhatsApp con sus compañeros de colegio.

Con la llegada de su primer smartphone se acrecienta notablemente su independencia. Ya tiene su perfil propio en alguna red social (incluso aunque tenga la edad mínima legal) y aunque interactúa con sus iguales, se trata de contactos esporádicos y se trata de una actividad más propia del ámbito del entretenimiento que de la socialización. Aparecen también los grupos de WhatsApp con familiares y gente de su entorno.

Inicialmente el smartphone del menor no suele tener ninguna contraseña y los padres pueden acceder a él con total libertad, posteriormente tiende a introducirse una contraseña o sistema de bloqueo que alguno de los padres (o los dos) conoce, hasta que la edad del adolescente es más avanzada y esgrime argumentos de privacidad para tener una contraseña propia, un factor que acrecienta notablemente su independencia.

El smartphone empieza a convertirse en un motivo de desencuentro familiar si los padres consideran que el adolescente lo consulta en exceso o si perciben que interfiere en la formación (cuando hace las tareas escolares o en época de exámenes), un hecho que se agravaría si se produce un descenso del rendimiento académico.

Es frecuente la restricción del uso del smartphone en ciertos momentos del día o en esos periodos más intensos de exigencia académica (con controles o exámenes) y el teléfono móvil se convierte en una herramienta de castigo, momento que algunos expertos consideran puede ser el germen de patologías posteriores o cuadros de ansiedad, si existe una excesiva recurrencia por parte de los padres a convertir el smartphone en un instrumento manipulativo con el que conseguir que sus hijos tengan el comportamiento que ellos desean.

\subsection{Papel de la familia}

Los menores manifiestan sentirse completamente acompañados en sus primeros pasos en el entorno digital. Si bien adquieren las destrezas en el manejo de los dispositivos de forma temprana, los padres suelen ser los primeros responsables en incorporar a sus hijos a la vida digital y tienden a ser sus primeros formadores. Los padres suelen ser los primeros que autorizan, crean (incluso a una edad en la que no está permitido legalmente) y supervisan sus primeros perfiles en redes sociales.

En esas fases iniciales los adolescentes también recurren a sus padres cuando tienen consultas o perciben que sucede algo inusual, como que les contacte alguien que no conocen. Es frecuente que en la primera etapa de la adolescencia los menores se muestren orgullosos de la confianza de sus padres y lo expresan mediante comentarios del tipo: "mis padres confían muchísimo en mí”, "a mis padres les cuento todo".

Tienden a hacer caso a los consejos que les dan sus padres, relativos a la información, fotografías, vídeos o datos que publican y comparten en redes sociales, si bien esto parece fluctuar en función de los hábitos que los menores observan en sus progenitores. Si sus padres son más activos en redes sociales, parece que los menores encuentran menos barreras para su propia actividad en el entorno digital.

Los padres tienden a insistir en las medidas de seguridad y a ser muy vigilantes. Con el paso del tiempo este control o vigilancia paterno empieza a resultar excesivo para algunos adolescentes y entre ellos se suelen compartir fórmulas para esquivar este control: desde la creación de perfiles que sus padres desconocen, pasando por aprovechar las opciones que permiten las aplicaciones para que sea más complejo seguir su actividad (como ocultar la hora de su última consulta de WhatsApp), hasta introducir una contraseña en su dispositivo que solo conozcan ellos.

A tenor de lo que manifiestan los adolescentes, el control o vigilancia que realizan los padres de sus hábitos y el tiempo que dedican al smartphone es muy diferente durante el curso académico y en especial en periodo de exámenes respecto al que se realiza en los periodos vacacionales. La supervisión en esta época del año es, según lo que comentan, prácticamente inexistente. 


\section{Conclusiones}

De los resultados obtenidos, puede concluirse que las redes sociales se convierten en la principal vía de escape para combatir el aburrimiento entre los adolescentes y destacan, por una de sus particularidades, la posibilidad de conectar con personas diferentes a las habituales en su círculo de contactos y de cualquier lugar del mundo.

Los adolescentes consideran que las redes sociales tienden cada vez a ser más completas por las posibilidades de uso que presentan. El uso particular que otorgaban a alguna de ellas por ciertas utilidades concretas con que no contaban las otras es cada vez menos frecuente. Su predilección por Instagram tiene como fundamento más reiterado en sus discursos la versatilidad de las alternativas que implementa y la mayoría manifiesta que toda la interacción con sus iguales podrían realizarla solo a través de esta red social. No se ha detectado, en la línea de lo expresado por Madden et al. (2013: 26), que interactuar lejos de la mirada de los adultos sea un factor que les lleve a usar Instagram u otras redes distintas de Facebook, sino más bien observar y estar informado de lo que hacen sus contactos o interactuar con ellos, pautas definidas en el estudio de Prades \& Carbonell (2016: 32), así como el hallazgo nuevo detectado en este estudio referente a que la integración de servicios en una misma red social supone un atractivo para el público adolescente a decantarse por ella.

Con la excepción de WhatsApp, los adolescentes suelen tener entre sus seguidores o contactos en sus redes sociales a personas con las que no han tenido un contacto real. Este hecho es, ya de por sí, un factor de riesgo, porque permite la interacción con usuarios que no conocen en su vida fuera del social media.

Como vulnerabilidad cabe destacar alguno de los inconvenientes asociados a toda exposición o participación en un espacio público: la posibilidad de recibir críticas (que pueden ser muy agresivas, injustas e incluso maliciosas) realizadas por personas que conocen en su vida diaria, pero también por otros individuos, lo que no necesariamente minimiza el impacto que les causa y cómo les afecta.

También se ha detectado la necesidad que tienen de recibir un apoyo o refuerzo positivo con cierta inmediatez cuando realizan algunas publicaciones como una fotografía, por ejemplo. El adolescente manifiesta sentir algún grado de ansiedad si no recibe de forma instantánea "likes" o "me gusta" que respalden el interés o la aceptación de su publicación, un factor asociado a esa conexión permanente (Del Barrio, 2014: 564).

Aunque reconocen consultar y seguir a los denominados influencers, no son ajenos a que las opiniones de estos atienden, en ocasiones, a criterios comerciales o de otra índole y que, en ocasiones, muestran conductas de dudosa ejemplaridad, por lo que se muestran también críticos hacia ellos y no tienen una actitud de seguidismo indiscriminada, lo que coincide con las conclusiones del estudio de Aran-Ramsport et ál. (2018: 78-79).

Con respecto a la seguridad, tienden a considerar que se sienten seguros usando las redes sociales. El respeto a ciertas pautas de protección básicas como el control de los contactos que aceptan y prestar atención a qué tipo de contenido comparten les da tranquilidad. La posibilidad de bloquear o incluso de denunciar a través de las propias redes sociales (estos son sus principales mecanismos de protección) los perfiles de usuarios que les molestan o incordian, les da tranquilidad y confianza. Estas interacciones negativas que han tenido no parece condicionar su percepción del riesgo, lo que coincide con lo concluido por De-Frutos \& Marcos (2017: 93).

Por otra parte, no se ha detectado que las chicas se manifiesten más preocupadas que los chicos por la seguridad en las redes sociales, tal y como exponían Rial et al. (2014: 650). Aunque se haya percibido que las chicas son más interpeladas por desconocidos que los chicos, estos también se muestran cautos con sus acciones y la menor preocupación que muestran algunos adolecentes parece estar relacionada con su menor exposición pública, no por una cuestión de género.

Los adolescentes manifiestan, de forma generalizada, que el acompañamiento de los padres es más intenso al 
inicio de su andadura en el entorno digital, tanto en lo relativo a consultar su smartphone como las acciones que realiza en él, las redes sociales en las que tiene dado de alta un perfil, los contactos que tiene en ellas, así como los tiempos de consumo. Con el paso del tiempo, dicho control se relaja y se ha detectado que existe una suma de factores que desencadena este menor control parental: la capacidad persuasiva de los adolescentes, que esgrimen argumentos de confianza a medida que se hacen mayores, para que se rebaje el control y las menores habilidades tecnológicas de los padres, tanto en lo relativo a ciertas redes sociales de las que no son usuarios como en lo relativo al manejo de los dispositivos, cada vez más sofisticados.

Respecto al uso del teléfono móvil, dispositivo de preferencia entre los adolescentes, se concluye que existe un excesivo contraste en la vigilancia y regulación del uso del smartphone en momentos concretos del calendario escolar (como las épocas de evaluación) si se compara con una amplia libertad que se les concede en los periodos vacacionales.

Entre las limitaciones de este estudio cabe señalar el hecho de tratarse de un estudio cualitativo, que por un lado permite conocer las tendencias en el uso y percepciones de los adolescentes sobre el tema, pero no conocer con exactitud la extensión de estas experiencias. Asimismo, cabe resaltar el hecho de recoger una mayor variedad de variables estructurales de la muestra para describir las opiniones de la población española, y un solo grupo para recoger la opinión de los adolescentes colombianos. Lo que implica la necesidad de contrastar las conclusiones obtenidas con un mayor número de grupos y de características más variadas en el contexto colombiano.

Este estudio ha recopilado las principales tendencias en las opiniones y percepciones de los adolescentes con respecto al uso de las redes sociales y del smartphone. Estas tendencias servirán de base para el diseño de una encuesta poblacional en profundidad sobre estos temas.

\subsection{Discusión}

La versatilidad de Instagram, tanto en su uso a través del smartphone como mediante el ordenador u otro dispositivo, la incorporación de multiplicidad de opciones (historias, mensajes, llamadas, compartir fotos y vídeos, etc.) que satisfacen a los jóvenes y la presencia de adultos en Facebook, son condicionantes que pueden explicar que Instagram se esté convirtiendo en la red social favorita del público adolescente en España. Sin embargo, aunque los adolescentes colombianos también manifiestan cierta predilección por Instagram, también tienen perfiles en Facebook y, en algún caso puntual, esta última sigue siendo su red preferida. Nuestra investigación confirma, por tanto, esas tendencias diferenciales halladas en estudios previos (Prades \& Carbonell 2016: 35; TigoUne 2018: 15). Los adolescentes tienden a ser receptivos a redes sociales con elementos innovadores (como en su momento fue Snapchat o actualmente Tik Tok) pero su preferencia por un social media más versátil e integrador, como Instagram, parece constatada.

Aunque no se pueda generalizar una diferencia de hábitos entre los adolescentes españoles y colombianos, a tenor del único grupo de discusión realizado en Colombia, sí quedó patente para los investigadores que los adolescentes colombianos que participaron en la investigación tenían un conocimiento más exhaustivo de las diferentes redes sociales y su diferente potencial, manifestaron unas inquietudes que en España serían propias de jóvenes con mayor edad e incluso al terminar la propia acción investigativa, siguieron libremente debatiendo en el foro creado manifestando un interés por alguno de los temas que habían surgido en el grupo de discusión. En ninguno de los grupos de discusión realizado con adolescentes españoles se manifestó un interés por continuar el debate cuando los investigadores daban por concluida la sesión.

En este estudio se ha manifestado que el poder divulgador y amplificador de cualquier situación de conflicto en el entorno digital provoca que los adolescentes se sientan más vulnerables, ya que cualquier incidente o burla pueden degenerar en problemas como la pérdida de autoestima. Quizás los menores recursos para la 
socialización o asimilación de la crítica que tiene un adolescente respecto a las herramientas de asertividad que puede poseer un adulto acrecienten esta percepción de vulnerabilidad. Por tanto, son los adolescentes que más se exponen, los más vulnerables. Anteriores estudios han situado en esta posición a las chicas ya que han constatado que su nivel de exposición es mayor (Alonso-Ruido et al., 2015: 56) y con mayor implicación al compartir sentimientos y material más personal (Alonso-Ruido et al., 2015: 55; Sabater \& Fernández 2015: 240246) de modo que el riesgo es potencialmente mayor si se compara con el tipo de crítica que puede recibir quien comparte otro contenido con menor implicación personal (como un vídeo musical o de humor).

A pesar de que los padres son quienes les introducen en el uso de las redes sociales, han manifestado de forma generalizada que son también estos quienes les retiran el smartphone en época de estudio. Esta tendencia parece estar relacionada con la distracción que puede provocar este dispositivo y la consulta reiterada de las redes sociales, lo que contrasta con lo "aburrido" que, según los adolescentes, resulta el periodo de estudio.

Por otra parte, durante las vacaciones, el smartphone es un elemento que entretiene al adolescente, especialmente en el periodo en que los padres continúan con sus obligaciones laborales y el menor ya no tiene que asistir a clases, lo que puede suponer un cierto alivio para los padres que no disponen de tanto tiempo libre como los jóvenes. No obstante, una excesiva relajación paterna puede incidir en el fomento de conductas que luego son complicadas de erradicar (como el tiempo excesivo de dedicación al teléfono móvil) o que, por combatir el aburrimiento, los adolescentes se sobreexpongan en exceso en sus perfiles de redes sociales.

Este trabajo de investigación se engloba en el proyecto "Redes sociales, adolescentes y jóvenes: convergencia de medios y cultura digital", referencia CSO2016-74980-C2-2-R, financiado por el Ministerio de Economía, Industria y Competitividad (España) dentro del Plan Nacional I+D+i. Plazo de ejecución del 30 de diciembre de 2016 al 29 de diciembre de 2020.

\section{Referencias bibliográficas}

Almansa, A., Fonseca, O. \& Castillo A. (2013). Redes sociales y jóvenes. Uso de Facebook en la juventud colombiana y española. Comunicar, 40(XX), 127-135. http://dx.doi.org/10.3916/C40-2013-03-03

Alonso-Ruido, P., Rodríguez-Castro, Y., Lameiras-Fernández, M. \& Carrera Fernández, M. V. (2015). Hábitos de uso en las redes sociales de los y las adolescentes: análisis de género. Revista de Estudios e Investigación en Psicología y Educación, 13, 054-057. https://doi.org/10.17979/reipe.2015.0.13.317

Álvarez, E., Heredia, H. y Romero, M. F. (2019). La Generación Z y las Redes Sociales. Una visión desde los adolescentes en España. Revista Espacios. 40(20), 9-22. Recuperado de: https://www.revistaespacios.com/a19v40n20/a19v40n20p09.pdf

Aran-Ramsport, S., Fedele, M. \& Tarragó, A. (2018). Las funciones sociales de los youtubers y su influencia en la preadolescencia. Comunicar, 57(XXVI), 71-80. https://doi.org/10.3916/C57-2018-07

Atuesta Reyes, J. D. (2014). Aspectos conceptuales sobre la enseñanza de las competencias científicas y tecnológicas en la educación básica. Páginas, 94, p. 63-74. Recuperado de: https://bit.ly/3hFklkX

Cantón Mayo, I., Arias Gago, A. R. \& Ruth Pinto, A. (2019). Acceso no regulado a internet y autopercepción de su imagen en las redes sociales. Etic@net: Revista científica electrónica de Educación y Comunicación en la Sociedad del Conocimiento, 19(1), 174-209. Recuperado de: https://bit.ly/32X8Zdu

Chóliz, M., Villanueva, V. \& Carmen Chóliz, M. (2009). Ellas, ellos y su móvil: uso, abuso (¿̇y dependencia?) del teléfono móvil en la adolescencia. Revista Española de Drogodependencias, 34(1), 74-88. Recuperado de: https://bit.ly/3g4GcHv 
Corredor, J. A., Humberto Pinzón, O. \& Marcela Guerrero, R. (2011). Mundo sin centro: cultura, construcción de la identidad y cognición en la era digital. Revista de Estudios Sociales, 40, 44-56. Recuperado de: https://bit.ly/3hKXN81

De-Frutos, B, \& Marcos, M. (2017). Disociación éntrelas experiencias negativas y la percepción de riesgo de las redes sociales en adolescentes. El profesional de la información, 26(1), 88-96.

https://doi.org/10.3145/epi.2017.ene.09

De la Fuente Prieto, J., Lacasa Díaz, P. \& Martínez-Borda, R. (2019). Adolescentes, redes sociales y universos transmedia: la alfabetización mediática en contextos participativos. Revista Latina de Comunicación Social, 74, 172-196. https://doi.org/10.4185/RLCS-2019-1326

Del Barrio, A. \& Ruiz, I. (2017). Hábitos de uso del whatsapp por parte de los adolescentes. International Journal of Development and Educational Psychology 1(1), 23-30.

https://doi.org/10.17060/ijodaep.2017.n1.v2.915

Del Barrio, A. (2014). Los adolescentes y el uso de los teléfonos móviles y los videojuegos. International Journal of Development and Educational Psychology 1(3), 563-570.

http://dx.doi.org/10.17060/ijodaep.2014.n1.v3.536

Espinar, E. \& López, C. (2009). Jóvenes y adolescentes ante las nuevas tecnologías: percepción de riesgos. Athenea Digital, 16, 1-20. Recuperado de: https://bit.ly/39vnSVw

García del Castillo J. A., García del Castillo-López, A., Dias, P. C. \& García-Castillo, F. (2019). Conceptualización del comportamiento emocional y la adicción a las redes sociales virtuales. Salud y Drogas 19(2), 173-181. Recuperado de: https://bit.ly/39xUe1W

García Jiménez, A., Catalina García, B. \& Cruz López de Ayala, M. (2016). Adolescents and YouTube: Creation, participation and consumption. Prisma Social 1, 60-89. Recuperado de: https://bit.ly/32YISnF

García Jiménez, A. \& Montes Vozmediano, M. (2020). Subject matter of videos for teens on YouTube. International Journal of Adolescence and Youth, 25(1), 63-78. https://doi.org/10.1080/02673843.2019.1590850

Garmendia, M., Jiménez, E., Casado, M. A. \& Mascheroni, G. (2016). “Net Children Go Mobile: Riesgos y oportunidades en internet y el uso de dispositivos móviles entre menores españoles (2010-2015)". Madrid: Red.es/UPV. Recuperado de: https://bit.ly/303sChX

Gil, F., Del Valle, G., Oberst, U. \& Chamarro, A. (2015). Nuevas tecnologías ¿Nuevas patologías? El smartphome y el fear of missing out. Aloma: Revista de Psicología, 33(2): 77-83. Recuperado de: https://bit.ly/2P6yErP

Giménez, A. M., Luengo, J. A. y Bartrina, M. J. (2017). ¿Qué hacen los menores en internet? Usos de las TIC, estrategias de supervisión parental y exposición a riesgos. Electronic Journal of Research in Educational Psychology, 15(3): 533-552. http://dx.doi.org/10.14204/ejrep.43.16123

Golpe, S., Gómez, P., Harris, S. K., Braña, T., \& Rial, A. (2017). Diferencias de sexo en el uso de internet en adolescentes españoles. Psicología Conductual, 25(1), 129-146. Recuperado de: https://bit.ly/3hEJSQM

Lenhart, A., Smith, A., Anderson, M., Duggan, M. \& Perrin, A. (2015). Teens, Technology and Friendships. Washington: Pew Research Center. Recuperado de: https://pewrsr.ch/3eZFOZC 
López-Sánchez, C. \& García del Castillo, J. A. (2017). La familia como mediadora ante la brecha digital: repercusión en la autoridad. Revista Colombiana de Ciencias Sociales, 8(1), 101-117. https://doi.org/10.21501/22161201.1928

Madden, M., Lenhart, A., Cortesi, S., Gasser, U., Duggan, M., Smith, A., \& Beaton, M. (2013). Teens, Social Media and Privacy. Washington: Pew Research Center. Recuperado de: https://pewrsr.ch/2P2OMLb

Malander, N. M. (2019). Adicciones tecnológicas en adolescentes: relación con la percepción de las prácticas parentales. Drugs and Addictive Behavior, 4(1), 25-45. Recuperado de: https://bit.ly/2P01Cd5

Marcelino Mercedes, G. V. (2015). Migración de los jóvenes españoles en redes sociales. De Tuenti a Facebook y de Facebook a Instagram. La segunda migración. Icono14, 13, 48-72.

http://dx.doi.org/10.7195/ri14.v13i2.821

Martín Nieto, R., Vizcaíno-Laorga, R. \& Pastor Ruiz, Y. (2017). “La participación social, los usos mediáticos y los riesgos de los adolescentes y jóvenes a través de Internet". En El fin de un modelo de política, Herrero, J. \& Trenta, T., (coords.), 397-435. Tenerife: Universidad de La Laguna. Recuperado de: https://bit.ly/2P01HOn

Martínez, E., García, A. y Sendín, J. C. (2013). Percepción de riesgos en la red por los adolescentes en España: Usos problemáticos y formas de control. Anàlisi: Quaderns de comunicació i cultura, 48, 111-130. https://doi.org/10.7238/a.v0iM.1969

Martínez, E., Catalina, B., \& López de Ayala, M. C. (2019). Smartphone, menores y vulnerabilidades. Revisión de la literatura. Revista Mediterránea de Comunicación, 10(2), 257-268. https://www.doi.org/10.14198/MEDCOM2019.10.2.5

Mejía Navarrete, J. (2000). El muestreo en la investigación cualitativa. Investigaciones sociales 4(5), 165-180. https://doi.org/10.15381/is.v4i5.6851

Merritt, M. (2008). Family Online Safety Guide. Recuperado de: https://bit.ly/39vR5j6

Montes-Vozmediano, M., García-Jiménez, A. \& Menor-Sendra, J. (2018). Teen videos on YouTube: Features and digital vulnerabilities. Comunicar, 54(1), 61-69. https://www.doi.org/10.3916/C54-2018-06

Moreno López, N. M., González Robles, A. C., Torres Gómez, A. C. y Araya Hernández, J. (2017). Alfabetización digital a padres de familia en el uso de las redes sociales. Alteridad, 12(1), 8-19.

http://doi.org/10.17163/alt.v12n1.2017.01

Pastor, Y., Martín, R. y Montes, M. (2019). Patrones de uso, control parental y acceso a la información de los adolescentes en la red. Estudios sobre el Mensaje Periodístico, 25(2), 995-1012. https://dx.doi.org/10.5209/esmp.64821

Pérez, V., Pastor, Y. \& Abarrou, S. (2018). Los youtubers y la construcción de la identidad adolescente. Comunicar, 55, 61-70. https://doi.org/10.3916/C55-2018-06

Prades, M. \& Carbonell, X. (2016). Motivaciones sociales y psicológicas para usar Instagram. Media Literacy \& Gender Studies, 5(9), 27-36. Recuperado de: https://bit.ly/2P1YA8c

Ramos, I., López, M. C. \& Torrecillas, T. (2018). Percepción de riesgo online en jóvenes y su efecto en el comportamiento digital. Comunicar, 56(XXVI), 71-79. https://doi.org/10.3916/C56-2018-07 
Rial, A., Gómez, P., Braña, T. \& Varela, J. (2014). Actitudes, percepciones y uso de Internet y las redes sociales entre los adolescentes de la comunidad gallega (España). Anales de Psicología, 30(2), 642-655. http://dx.doi.org/10.6018/analesps.30.2.159111

Rodríguez, L. \& Magdalena, J. R. (2016). Perspectiva de los jóvenes sobre seguridad y privacidad en las redes sociales. Icono14 14, 24-49. http://dx.doi.org/10.7195/ri14.v14i1.885

Sabater, C. \& Bingen Fernández, J. (2015). No, sin mi móvil. Diferencias de género y uso de las nuevas tecnologías. Icono 14, 13(1), 208-246. https://doi.org/10.7195/ri14.v13i1.722

Santana-Vega, L., Gómez-Muñoz, A. M., Feliciano-García, L. (2019). Uso problemático del móvil, fobia a sentirse excluido y comunicación familiar de los adolescentes. Comunicar 59(XXVII), 39-47. https://doi.org/10.3916/C59-2019-04

Sendín, J. C., Gaona, M. C. \& García, A. (2014). Nuevos medios: usos comunicativos de los adolescentes. Perspectivas desde los nativos digitales. Estudios sobre el Mensaje Periodístico, 20(1), 265-283. https://doi.org/10.5209/rev_ESMP.2014.v20.n1.45231

Tejada Garitan, E., Castaño Garrido, C. \& Romero Andonegui, A. (2019). Los hábitos de uso en las redes s sociales de los preadolescentes. RIED. Revista Iberoamericana de Educación a Distancia, 22(2), 119-133. http://dx.doi.org/10.5944/ried.22.2.23245

TigoUne (2018). "Estudio sobre riesgos y oportunidades en el uso de Internet: Informe Nacional". Tigo (Colombia). Recuperado de: https://bit.ly/2X2Y4Lt

Vizcaíno-Laorga, R., Montes Vozmediano, M. \& De La Torre, P. (2017). Tocando la realidad en la producción de contenidos informativos, rutinas profesionales y participación en entornos digitales. Prisma Social, 19, 192-225. Recuperado de: https://bit.ly/30VL7nO

Esta obra está bajo una Licencia Creative Commons Attribución-NoCommercial 4.0 International

\section{(cc) BY-NC}

